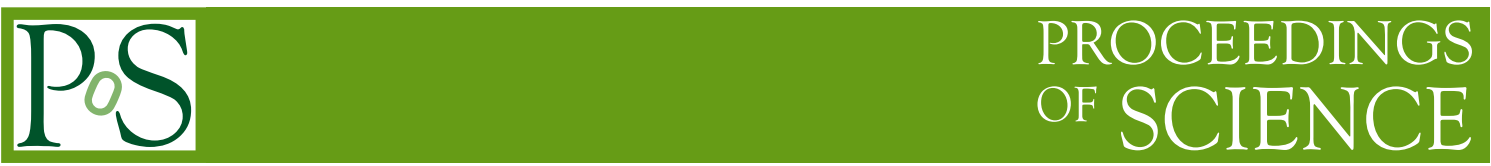

\title{
Galaxies as accelerators of cosmic rays
}

\author{
V. V. Uchaikin \\ Ulyanovsk State University, Ulyanovsk, Russia \\ E-mail: vuchaikin@gmail.com \\ R. T. Sibatov* \\ Ulyanovsk State University, Ulyanovsk, Russia \\ E-mail: ren_sib@bk.ru

\section{O. P. Harlova} \\ Ulyanovsk Higher Civil Aviation School (Institute), Ulyanovsk, Russia \\ E-mail: harlova.op@gmail.com
}

In the frame of large-scale structure of the Universe, cosmic rays (CRs) can be considered as a component of the intergalactic medium, which originate and accelerate inside galaxies, leave them, fill the intergalctic space and have possibility to enter other galaxies and continue acceleration. Multiple repeating, this process may, in principle, produce CRs with ultra-high energy. To estimate characteristics of this process, we consider a simple version, when a charged particle falls on a plane layer of ISM and is reflected from it. As is known, the diffusion approximation fails to get the time and path distributions, so we apply more proper tools for calculations. The time distribution is obtained via the analytical solution of the linearized Boltzmann equation, and the acceleration process is calculated by solving the integral reacceleration equation. In addition, our analysis takes a fractal (turbulent) properties of ISM into account in frame of the nonlocal CR transport theory. Numerical calculations show that CRs scattering and accelerating in other galaxies get a more hard spectrum and for this reason may be available for observation.

The 34th International Cosmic Ray Conference,

30 July- 6 August, 2015

The Hague, The Netherlands

\footnotetext{
${ }^{*}$ Speaker.
} 


\section{Introduction}

Considering the intergalactic space filled with cosmic rays, one can suppose that the galaxies may serves not only as generators of CRs but also as accelerators: entering the galaxies and walking in them, the CRs continue to raise their energy due to distributed reaccelerations on SNRs [1]. This phenomenon may play some role in solving such an important problem as the origin of extragalactic cosmic rays [2] or at least in estimation of CR spectrum in intra-cluster medium.

We are interested in energy (momentum) spectrum of CRs entering a half-space medium with momentum $p_{0}$ and leaving it through the same surface after a while. The simplest version of the problem looks like a specific statement of the Milne problem well-known in the astrophysics [3]: a particle enters the half-space with momentum $\mathbf{p}_{0}=p_{0} \vec{\Omega}_{0}$ and leaves it random time after with momentum $\mathbf{p}=p \vec{\Omega}$ (Fig.1). As opposed to initial use of the model for investigation of light propagation through planet atmospheres, we apply it to the problem of reflection (albedo) CRs from galaxies. Two principal specifics distinguish the second problem from the previous: acceleration on SNRs and large-scale heterogeneity of turbulent interstellar magnetic field. Our aim is to estimate a distributed acceleration role in forming albedo spectrum of galaxies. We simplify the problem reducing it to estimation of the albedo spectrum for semi-infinite interstellar medium with a plain boundary (that is we neglect by the curvature of the galaxy surface and by exit of particles through the side and opposite boundaries.

Solving à time-dependent problem is performed in two steps. First, we find the waiting time distribution $w(t)$. Here, $w(t) d t$ is the probability that the time spent by the particle, say, a proton, in the accelerating half-space $x>0$ falls between $t$ and $t+d t$. At the second step, we determine the albedo spectrum $f(p \mid t)$ conditioned by a fixed time $t$. The total spectrum is represented by integral

$$
f(p)=\int_{0}^{\infty} f(p \mid t) w(t) d t .
$$

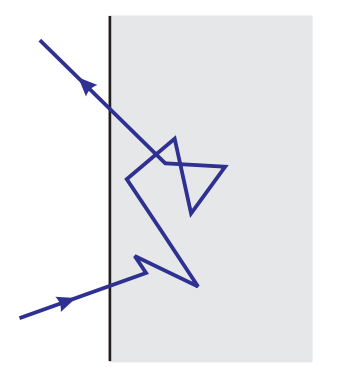

Figure 1: Schematic of the Milne problem.

\section{One-velocity CR-transport model}

Each of pdf's in right hand side of equality (1) obeys an own equation determining by the model accepted for description of the process. The classic transport model relating to the process in a uniformly homogeneous media (this term means the case when free paths of a walking particle 
are mutually independent and exponentially distributed) is described by the integro-differential Boltzmann equation [3].

The special kind of solution related to our problem has been obtained in [4]. An instantaneous isotropic point source acts at time $t=0$ at the surface $x=0$, and the albedo current on the boundary is observed:

$$
j_{x}(t)=\int_{-1}^{0} d \mu \int_{0}^{1} d \mu_{0} \psi\left(0, \mu, t ; \mu_{0}\right) .
$$

The resulting sought-for pdf $w(t)$ is expressed via the modified Bessel function $I_{1}(x)$ as follows

$$
w(t)=\frac{j_{x}(t)}{\int_{0}^{\infty} j_{x}(t) d t}=t^{-1} e^{-\sigma v t / 2} I_{1}(\sigma v t / 2) .
$$

We use Monte Carlo (MC) method for further calculation. Fig. 2 shows that its result are in agreement with analytical representation (3).

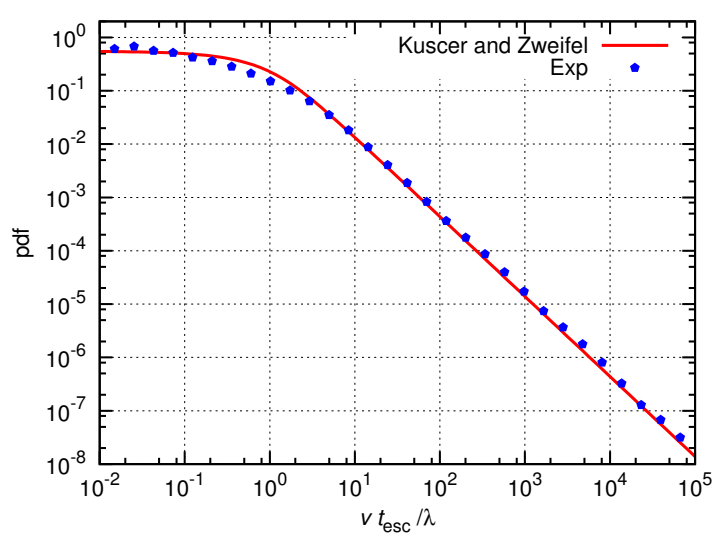

Figure 2: Distribution of escape times in case of random walk with exponential distribution of free path lengths.

\section{Why not a simple diffusion}

Observe that the problem of finding the albedo time distribution has no solution in frame of the ordinary diffusion theory. The diffusion equation

$$
\frac{\partial \rho}{\partial t}=D \frac{\partial^{2} \rho}{\partial x^{2}}
$$

results from the Boltzmann equation as a limit case $v \rightarrow \infty, l \rightarrow 0$ under condition $v l / 3 \rightarrow D<\infty$. As easily to see, under this condition solution (3) becomes delta-function: $w(t) \rightarrow \delta(t)$. Gaussian solution of Eq. (4) seems to be quite acceptable but it doesn't represent trajectories in an explicit form. Their patterns are revealed in frame of the Wiener representation of the Brownian motion which leads to some paradoxical conclusion: the particle moving according to Eq. (4) changes its motion direction infinite times during an arbitrarily short time interval. Starting its motion at the point of the boundary it immediately leaves it going to another half-space (Fig. 3). In order to find 
$w(t)$, one should disavow the diffusion model and come back to the Boltzmann level of transport interpretation involving the finite velocity and free path conceptions.

In contrast to the diffusion approximation, this approach produces correlations between displacements of a particle during non-overlapping time intervals.

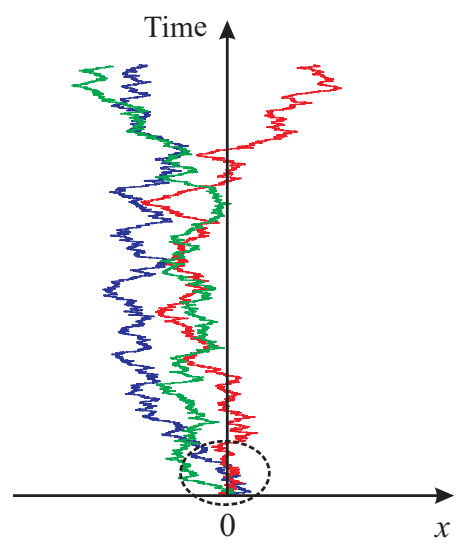

Figure 3: Trajectories of the Brownian motion.

\section{Why not the Boltzmann kinetics}

Nevertheless, it is not enough for adequate description of interstellar transport even in the guiding center approximation. The point is that operator

$$
L=\frac{\partial}{\partial t}+\mu v \frac{\partial}{\partial x}
$$

appears in the case of absence of any correlations between points of scattering. This is quite acceptable for propagation of particles through a rarefied gas of molecules distributed in space independently and uniformly. One can show that the free path $\xi$ of a particle between collisions in this medium is distributed according to the exponential law

$$
\operatorname{Prob}(\xi>x)=e^{-(\sigma / \mu) x}
$$

related to the operator (5). However, this distribution seems wrong for the points of scattering being located at special points of magnetic field lines. As a result, the scattering points are not independent but linked via long correlations. Moreover, the process takes place in a turbulent medium, so the correlations are rather of inverse power law kind than of exponential one:

$$
\operatorname{Prob}(\xi>x) \propto x^{-\alpha}, \quad \alpha>0, \quad x \rightarrow \infty .
$$

Observe that the first moment of $\xi$ exists only for $\alpha>1$, and the variance only for $\alpha>2$. Media of such kind are known as fractal ones, and many astrophysicists agree to consider that interstellar medium belongs to this type [9]. First applications of the model to CR propagation problems were made in [10] and [11].

Replacing pdf (6) by pdf (7) causes transformation of integro-differential equation into its fractional counterpart [5], containing fractional differential operator $L^{\alpha}$ instead of (5). We solve 



Figure 4: Distribution of escape time (left panel) and number of scattering (right panel) in a semi-infinite medium characterized by different distributions of free path length.

it by the MC method. Fig 4 shows the results of these calculations. In the exponential case, pdf $q(t)$ calculated by the MC method strictly coincides with analytical solution (3). To retrace its evolution with variation of $\alpha$, we represent time $t$ in terms of median value of free flight time. As one can see from Fig. 4, the increase in heterogeneity of the medium significantly enlarges the time spent by particles in the accelerated medium. In case of a homogeneous medium, would mean acceleration up to higher energy, but this is not the case. Our calculations show that the number of events accelerating particles are distributed according to inverse power law as well, but its index doesn't depend on $\alpha$ (Fig. 4, right panel). For this reason, the distribution of event number is a more appropriate characteristics of ISM for calculations of acceleration than the time distribution.

\section{Distributed reacceleration}

In contrast to the preceding problem, the reacceleration process concerns the behavior of particles in the momentum space. The Fermi model of successive interactions (collisions) of a charged particle with more or less localized inhomogeneities of the magnetic field (magnetic clouds) was generalized to strong shock waves in the supernovae remnants discussed by Berezhko and Krymsky in review [6] and realized in [1]. From probabilistic point of view, this process can be interpreted as a sequence of instantaneous random jumps from one point of the momentum space to another one at random times (random jump process). The momenta $\Delta \mathbf{p}_{i}$ acquired by the particle in these collisions are random and, even for their isotropic distribution, the point

$$
\mathbf{p}=\mathbf{p}_{0}+\Delta \mathbf{p}_{1}+\Delta \mathbf{p}_{2}+\Delta \mathbf{p}_{3}+\ldots,
$$

representing the particle in the momentum space moves away from the point (momentum) of the acceleration injection $\mathbf{p}_{0}$ similar to a Brownian particle; this behavior means the further acceleration (reacceleration) of the particle. However, only a certain fraction of the particles moving away from the center are accelerated. This high-energy component forms the interstellar CR-field. 
The increment of the momentum in the multiplicative model is proportional (in the statistical case) to the absolute value of the momentum $p^{\prime}$ of the particle coming into interaction,

$$
\Delta \mathbf{p}=p^{\prime} \mathbf{Q}, \quad \int_{|\Delta \mathbf{p}|>p} w\left(\Delta \mathbf{p} ; \mathbf{p}^{\prime}\right) d \Delta \mathbf{p} \propto\left(p / p^{\prime}\right)^{-\gamma}, p \rightarrow \infty .
$$

On the assumption that the distribution of the random vector $\mathbf{Q}$ is independent of $\mathbf{p}^{\prime}$, isotropic and has the form

$$
W\left(\mathbf{q} ; \mathbf{p}^{\prime}\right) d \mathbf{q}=(1 / 2) V(q) d q d \cos (\mathbf{q}, \mathbf{p})
$$

with

$$
V(q)=\gamma q^{-\gamma-1}, \quad \gamma>1,
$$

the following equation approximates this process [1]:

$$
\frac{\partial}{\partial t} f(p \mid t)=\sigma v\left\{\int_{1}^{\infty} \gamma q^{-\gamma-1} f(p / q \mid t) d q / q-f(p \mid t)\right\}+f_{0}(p) \delta(t) .
$$

In what follows, we assume $f_{0}(p)=\delta(p-1)$. Let us stress that this equation is derived on assumption that the acceleration takes place at each collision and the random distance between consecutive collisions is distributed as in Eq. (6). Accounting the turbulent character of the interstellar magnetic field by means of replacement of (6) by (7) leads to a fractional version of Eq. (10) obtained in [7].

For the reasons discussed above, we represent the solution of the fractional version through the event number distribution $w(n)$ interpreting the latter as a continuous distribution,

$$
f(p)=\int_{0}^{\infty} f(p \mid n) w(n) d n
$$

As one can understand from above, $f(p \mid n)$ is a pdf of random variable $\Pi_{n}=Q_{1} Q_{2} \ldots Q_{n}$,

$$
f(p \mid n) d p=\operatorname{Prob}\left(p<\Pi_{n}<p+d p\right)
$$

being none other than the product of mutually independent random variables $Q_{1}, Q_{2}, \ldots Q_{n}$ distributed according to the common law (9). At first glance, it may seem that this random variable obeys the lognormal distribution at large $n$, but it is not the case: the distribution of $\Pi_{n}$ follows the log-normal distribution only in the middle scale region, whereas its large deviations relating to cosmic rays preserve the inverse power tail up to a slowly varying factor (see investigation of this problem in [8]). Our calculations confirm this fact (Fig. 5). As a result, we obtain, that the albedo-particles momentum spectra from a fractal half-space accelerating the particles have the pdf of a power type with the index a little be more than 1 independently of the fractal index $\alpha$. This pdf can be represented by a universal curve (Fig 6).

Finally, we investigated behavior of the process when the free path of the particles increases with $p$ as $p^{\delta}$ and saw that in this case the domain of this universality narrows to $1<\alpha<2$ (Fig. 6).

As noted above, equations (2) and (10) describe the transport process as if it took place in a homogeneous medium where the random free paths between acceleration events have exponential 


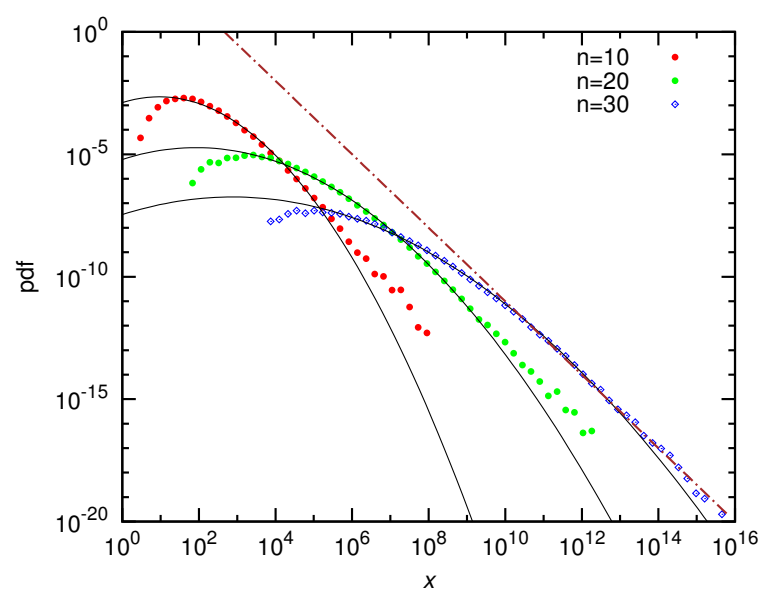

Figure 5: Distribution of the product of $n$ random multipliers distributed according to the power law, $p_{\xi}(x)=$ $\alpha x_{0} x^{-\alpha-1}, \alpha=1.5$.

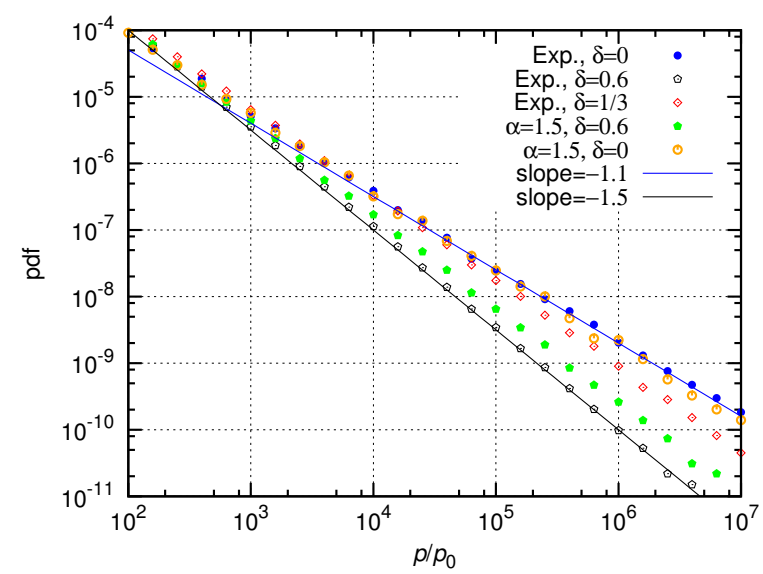

Figure 6: Momentum distributions in case of the exponential and power distributions of free path lengths, and $\delta=1 / 3$ and $\delta=0.6$.

distribution (6). In order to take into account the turbulent inhomogeneous we should pass from (6) to (7) in the momentum equation (10) as well, that is replace the first-order time-derivative by its fractional-order analog. Seeking its solution in the form of (11), we calculate the conditional pdf (12) and number event distribution $w(n)$ we found out:

1. In case of identical pdf of each free path, the distribution $w(n)$ is practically independent of the fractal index $\alpha \in(0,2)$.

2. Under this condition, the momentum distribution is represented by the universal power-type curve with an index, a little exceeding 1.

3. In case when free path length increases proportionally to $p^{\delta}$, the domain of this universality narrows to $1<\alpha<2$. 


\section{Acknowledgment}

Authors thank the Russian Foundation for Basic Research (project no. 13-01-00585) and the Ministry of Education and Science of the Russian Federation (2014/296) for financial support.

\section{References}

[1] A. Wandel, D. Eichler, J.R. Letaw, R. Silberberg, C.H. Tsao, Distributed reacceleration of cosmic rays.The Astrophysical Journal 316 (1987) 676.

[2] T. Enßlin. Extragalactic cosmic rays and magnetic fields: Facts and fiction.Journal of the Korean Astronomical Society 37, 5 (2004) 439.

[3] K. M. Case, P.F. Zweifel, Linear transport theory, Addison-Wesley Pub. Co Reading, Massachusetts (1967).

[4] I. Kuščer, P.F. Zweifel, Time-dependent one-speed albedo problem for a semi-infinite medium. Journal of Mathematical Physics, 6, 7 (1965) 1125.

[5] V.V. Uchaikin, R.T. Sibatov, R. T. Fractional Boltzmann equation for multiple scattering of resonance radiation in low-temperature plasma. Journal of Physics A: Mathematical and Theoretical, 44, 14 (2011) 145501.

[6] E.G. Berezhko, G.F. Krymskii, Acceleration of cosmic rays by shock waves.Physics-Uspekhi 31, 1 (1988) 27.

[7] V.V. Uchaikin, Fractional models of cosmic ray acceleration in the Galaxy.JETP Letters 92,4 (2010) 200 .

[8] D. Sornette, R. Cont, Convergent multiplicative processes repelled from zero: power laws and truncated power laws.Journal de Physique I 7, 3 (1997) 431.

[9] N. Sánchez, E.J. Alfaro, E. Pérez, The fractal dimension of projected clouds.The Astrophysical Journal 625, 2 (2005) 849.

[10] B.R. Ragot, J.G. Kirk, Anomalous transport of cosmic ray electrons. Astron. Astrophys. 327 (1997) 432.

[11] A.A. Lagutin, Y.A. Nikulin, V.V. Uchaikin, The "knee" in the primary cosmic rays spectrum as consequence of fractal structure of the galactic magnetic field. Preprint ASU-2000/4 (2000). 